\title{
Хроматографическая оценка влияния остарина и экдистерона на стероидный профиль мужчин и женщин
}

\author{
(C) 2020 Подольский И.И. ${ }^{1}$, Мочалова Е.С. ${ }^{2}$, Темердашев А.3. ${ }^{3}$ \\ ${ }^{1}$ ООО «Брукер», Российская Федераиия, Москва \\ ${ }^{2}$ Национальная антидопинговая лаборатория (институт) Московского государственного \\ университета имени М.В. Ломоносова, Москва \\ ${ }^{3}$ ФГБОУ ВО Кубанский государственный университет, Краснодар
}

Поступила в редакцию 1.06 .2020 г.

DOI: $10.17308 /$ sorpchrom.2020.20/2951

Селективные модуляторы андрогенных рецепторов относятся к новому классу допингагентов, позволяющих существенно повысить продуктивность спортсменов без проявления побочных эффектов, характерных для андрогенных анаболических стероидов. На сегодняшний день известен ряд методов их определения в биологических жидкостях человека, однако сведения об их влиянии на стероидный профиль человека практически отсутствуют. В представленной работе показано влияние употребления остарина на стероидный профиль мужчин и женщин. Исследование базируется на результатах качественного и количественного анализа более чем 10 тысяч проб мочи, не содержащих запрещенных ВАДА соединений, для получения статистически значимой репрезентативной выборки, 17 положительных женских и 13 мужских пробах, в которых идентифицированы остарин или его метаболиты, а также 532 мужских и 245 женских проб с подтвержденным экдистероном. Оценка содержания андрогенных стероидов проводилась с использованием валидированной методики газохроматографического анализа с тандемным масс-спектрометрическим детектированием в режиме множественного мониторинга реакций после дериватизации образцов смесью, содержащий дитиотреитол, йодид аммония и $\mathrm{N}$-метилтриметилсилилтрифторацетамид. Установлено, что употребление остарина приводит к существенному изменению содержания андрогенных стероидов у женщин, в то время как у мужчин подобный эффект не наблюдается. Отмечено, что употребление экдистерона не оказывает существенного влияния на содержание андрогенных стероидных гормонов ни у мужчин, ни у женщин. На основе рассмотренной выборки образцов, не содержащих соединений, входящих в Запрещенный список ВАДА, предложены более жесткие допустимые границы содержания андрогенных стероидов у женщин, не приводящие к существенному росту числа ложноположительных результатов. Применение данного подхода позволяет получить дополнительные косвенные признаки, указывающие на возможность применения спортсменом запрещенных препаратов, не прибегая к использованию дополнительных процедур и исследований с последующим использованием подтверждающих методов исследования в отношении подозрительных или предположительно положительных проб. Применение подобного комплексного подхода позволит существенно повысить надежность исследований и выявляемость положительных проб без увеличения времени и стоимости анализа.

Ключевые слова: ГХ-МС/МС, дериватизация, допинг, стероиды, остарин, экдистерон, стероидный профиль.

\section{Введение}

Селективные модуляторы андрогенных рецепторов (SARM) относятся к новому поколению допинг-агентов. Несмотря на постоянное пополнение перечня запрещенных соединений, они по-прежнему реализуются на рынке спортивного питания наравне с фитостероидами и другими биологически активными добавками. При- 
нимая во внимание тот факт, что для большинства из них не закончен полный цикл клинических испытаний, достаточно сложно предположить последствия их употребления как в краткосрочной, так и в долгосрочной перспективе [1-3].

На сегодняшний день известен ряд работ, посвященных определению ряда SARM и их метаболитов в биологических жидкостях человека. Остарин и андарин относятся к одним из первых коммерчески доступных и наиболее изученных представителей этого класса соединений [4-9]. Согласно последнему доступному отчету Всемирного Антидопингового Агентства (ВАДА) за 2018 год [10], количество выявляемых случаев употребления SARM ежегодно растет, из них более 58\% положительных проб приходится именно на обнаружение остарина и его метаболитов.

Очевидно, что влияние одних и тех же препаратов, стимулирующих или подавляющих выработку гормонов, может существенно отличаться у мужчин и женщин. Оценка этих изменений возможна только при наличии репрезентативной выборки и использовании базовых критериев, основанных на популяционных исследованиях. Работы подобного масштаба ранее проводились ВАДА в рамках развития направления, посвященного изучению стероидного профиля человека [11], а также установлению границ нормального содержания стероидов в биологических жидкостях человека.

На сегодняшний день известен ряд работ, посвященных хромато-массспектрометрическому определению широкого круга андрогенных стероидов [12-19] в биологических жидкостях человека, поскольку их содержание помимо допингконтроля может иметь клинико-диагностическое значение. Однако во избежание получения ложных результатов, необходимо принимать во внимание наличие соединений, способных оказывать влияние на биологический паспорт человека, в основе которого лежат гематологический и стероидный профиль человека. В настоящее время полученные в ходе мониторинга стероидного профиля данные используются для установления индивидуальных границ содержания стероидов в биологических жидкостях спортсмена с применением критерия Байеса, а наличие значимых выбросов в полученных результатах может являться основанием для проведения дополнительных исследований для установления факта употребления экзогенных стероидов [20, 21].

Принимая во внимание распространение селективных модуляторов андрогенных рецепторов [4-9] и фитостероидов [20-22], актуальным представляется изучение возможности расширения диагностической значимости стероидного профиля в целях изучение влияния остарина и, включенного в программу мониторинга 2020 года [23], экдистерона на стероидный профиль мужчин и женщин.

Целью данного исследования являлось хромато-масс-спектрометрическое определение андрогенных анаболических стероидов в моче с целью установления влияния употребления остарина и экдистерина на стероидный профиль человека с применением статистических методов анализа и введения более жестких критериев содержания андрогенных стероидов с целью косвенного установления факта их употребления.

\section{Экспериментальная часть}

Объект исследования. Результаты получены путем анализа рандомизированной и анонимизированной выборки образцов, поступавших для проведения научноисследовательских работ в течение нескольких лет во ФГУП «Антидопинговый центр» (позднее - Национальная антидопинговая лаборатория (институт) Московского государственного университета имени М.В. Ломоносова) от мужчин и женщин 
в возрасте от 18 до 40 лет, информированных о целях проведения исследования. Среди полученных образцов присутствовали 30 (13 мужских и 17 женских) с положительным результатом исследования на остарин (и/или его метаболиты) и 778 проб (532 мужских и 246 женских) с выявленным употреблением экдистерона.

Реактивы и реагенты. Стандартные образцы стероидных гормонов (тестостерон, метилтестостерон, этиохоланолон, андростерон, эпитестостерон, $5 \alpha$-андростан$3 \alpha, 17 \beta$-диол, $5 \beta$-андростан-3 $\alpha, 17 \beta$-диол, использованных в работе, чистотой $>99 \%$ были приобретены у компании Cerilliant (США), дигидрофосфат калия, дитиотреитол (ДТТ), гидрокарбонат калия чистотой, йодид аммония $>99 \%$ приобретены у Sigma-Aldrich (США). Для проведения работ также использовались диэтиловый эфир (99\%, Fisher Chemical), гелий 6.0 (Линде газ Рус, Россия), аргон 6.0 (Линде газ Рус, Россия); $\beta$-глюкуронидаза E. Coli K12 (Roche, Германия), МСТФА (>99\%, MachareyNagel, Германия), безводный сульфат натрия (Sigma-Aldrich, CША).

Оборудование. Для проведения анализа использовался газовый хроматограф Thermo Scientific Trace-1310, оснащенный капиллярной колонкой Agilent HP Ultra-1 (17 м×0.2 мм, толщина пленки неподвижной жидкой фазы - 0.11 мкм) со следующей температурной программой: начальная температура термостата колонок $-180^{\circ} \mathrm{C} \mathrm{c}$ последующим нагревом до $235^{\circ} \mathrm{C}$ со скоростью $4^{\circ} \mathrm{C} /$ мин с последующей интенсификацией скорости нагрева термостата до $20^{\circ} \mathrm{C} /$ мин до достижения температуры 310 ${ }^{\circ} \mathrm{C}$. Общее время анализа составило 22 минуты. Масс-спектрометрическое детектирование осуществлялось с применением тандемного масс-спектрометрического детектора Thermo Scientific TSQ Quantum XLS Ultra. Задержка на время элюирования растворителя - 4.25 мин. Температура инжектора $300{ }^{\circ} \mathrm{C}$, температура переходной линии $300^{\circ} \mathrm{C}$, газ-носитель - гелий, скорость потока газа-носителя через колонку $0.6 \mathrm{~cm}^{3} /$ мин, температура источника ионизации $-250^{\circ} \mathrm{C}$, давление газа-мишени (аргона) в ячейке соударений - 1.5 мТорр.

Пробоподготовка. К аликвоте мочи объемом $3 \mathrm{~cm}^{3}$ добавляли $1 \mathrm{~cm}^{3}$ фосфатного буферного раствора (pH 6.4), содержащего 30 мкл $\beta$-глюкуронидаза E. Coli K12 и внутренний стандарт (ВС) (метилтестостерон с концентрацией $150 \mathrm{нг} / \mathrm{cm}^{3}$ ) с последующей инкубацией при $57{ }^{\circ} \mathrm{C}$ в течение 40 мин, после чего к охлажденному до комнатной температуры гидролизату добавляли $1 \mathrm{~cm}^{3}$ карбонатного буфера (pH 10), 1.5 г безводного сульфата натрия и $5 \mathrm{~cm}^{3}$ диэтилового эфира, тщательно перемешивали в течение 10 мин, центрифугировали 5 мин при 4000 об/мин, после чего вымораживали водный слой в криостате при $-30^{\circ} \mathrm{C}$ и декантировали органическую фазу в чистые пробирки. Эфирный экстракт упаривали в твердотельном нагревателе при температуре $70^{\circ} \mathrm{C}$, охлаждали до комнатной температуры, вносили 50 мкл дериватизирующего раствора (20 мг ДТТ и 15 мг $\mathrm{NH}_{4} \mathrm{I}$ на $10 \mathrm{~cm}^{3}$ МСТФА) и инкубировали 20 мин при $70^{\circ} \mathrm{C}$. После окончания дериватизации охлажденную пробу переносили в стеклянную виалу с силанизированной вставкой объемом 100 мкл.

\section{Обсуждение результатов}

Анализ полученных проб осуществляли с использованием метода газовой хроматографии с тандемным масс-спектрометрическим детектированием. Применение ДТТ в составе дериватизирующего агента обусловлено необходимостью енолизации кето-группы для всех маркеров стероидного профиля, что позволяет получить ди-ТМС производные, что, в свою очередь, улучшает хроматографические свойства аналитов, а также существенно повышает чувствительность и селективность их дальнейшего определения с использованием режима множественного мониторинга реакций (ММР). 
Основные параметры для проведения качественного и количественного анализа исследуемых образцов приведены в табл. 1.

Таблица 1. Времена удерживания, ионы-прекурсоры, ионы-продукты и энергии соударений аналитов.

Table 1. Retention time, precursor ions, product ions, and collision energies of the analytes.

\begin{tabular}{|c|c|c|c|c|}
\hline Соединение & $\begin{array}{c}\text { Ион- } \\
\text { прекурсор, } \\
m / z \\
\end{array}$ & $\begin{array}{c}\text { Ион- } \\
\text { продукт, } \\
\mathrm{m} / \mathrm{z} \\
\end{array}$ & $\begin{array}{l}\text { Энергия со- } \\
\text { ударений, эВ }\end{array}$ & $\begin{array}{l}\text { Время удер- } \\
\text { живания, мин }\end{array}$ \\
\hline $\begin{array}{c}\text { Метилтестостерон ди-ТМС } \\
\text { (ВС) }\end{array}$ & $446^{*}$ & 301 & 20 & 13.8 \\
\hline \multirow{3}{*}{ Андростерон ди-ТМС } & 434* & 239 & 18 & \multirow{3}{*}{10.2} \\
\hline & 434 & 329 & 13 & \\
\hline & 434 & 419 & 9 & \\
\hline \multirow{3}{*}{ Этиохоланолон ди-ТМС } & 434 & 419 & 8 & \multirow{3}{*}{10.4} \\
\hline & 434 & 329 & 13 & \\
\hline & $434^{*}$ & 239 & 16 & \\
\hline \multirow{3}{*}{ Тестостерон ди-ТМС } & 417 & 301 & 10 & \multirow{3}{*}{12.4} \\
\hline & 432 & 417 & 12 & \\
\hline & $432 *$ & 209 & 14 & \\
\hline \multirow{4}{*}{ Эпитестостерон ди-ТМС } & 417 & 327 & 9 & \multirow{4}{*}{11.7} \\
\hline & 432 & 327 & 14 & \\
\hline & $432 *$ & 209 & 11 & \\
\hline & 432 & 417 & 11 & \\
\hline \multirow{4}{*}{$\begin{array}{c}5 \alpha \text {-андростан-3 } \alpha, 17 \beta \text {-диол } \\
\text { ди-ТМС }\end{array}$} & 256 & 227 & 10 & \multirow{4}{*}{10.5} \\
\hline & 256 & 213 & 12 & \\
\hline & 256 & 199 & 9 & \\
\hline & $256^{*}$ & 185 & 12 & \\
\hline \multirow{3}{*}{$\begin{array}{c}5 \beta \text {-андростан-3 } \alpha, 17 \beta \text {-диол } \\
\text { ди-ТМС }\end{array}$} & 256 & 199 & 9 & \multirow{3}{*}{10.6} \\
\hline & $256^{*}$ & 185 & 14 & \\
\hline & 331 & 241 & 11 & \\
\hline
\end{tabular}

* - переход для количественного анализа

Согласно регламенту ВАДА о критериях идентификации [26] для проведения качественного и количественного анализа рекомендуется использование нескольких ММР-переходов с целью повышения надежности получаемых данных. В случае применения внутреннего стандарта использовался только один переход для количественного анализа, поскольку подтверждение его наличия в образце не требовалось.

Полученные в ходе анализа результаты проверяли на соответствие критериям ВАДА (табл. 2) с последующим анализом общего массива данных для выявления однородности с результатами популяционных исследований.

Как видно из рис. 1, соотношение тестостерон/эпитестостерон (T/E) хорошо коррелирует с описанными ВАДА данными, наблюдается идентичное бимодальное распределение в схожих пределах, что также свидетельствует о корректности полученных результатов. Для андростерона (рис. 2) также наблюдается нормальное распределение.

Принимая во внимание значимый массив полученных данных, целесообразным представляется оценка влияния употребления остарина и экдистерона на стероидный профиль путем оценки содержания андрогенных стероидов в пробах мочи, в которых ранее было подтверждено наличие экдистерона, остарина или его метабо- 
литов. Как видно из рис. 3, при уменьшении окна допустимых пороговых значений по ряду показателей, видно, что употребление остарина приводит к увеличению содержания андрогенов у женщин.

Таблица 2. Допустимые значения содержания андрогенных стероидов и их соотношения в моче у мужчин и женщин в соответствии с критериями ВАДА.

Table 2. Permissible content of androgenic steroids and their ratio in male and female urine according to the requirements of WADA.

\begin{tabular}{|c|c|c|}
\hline \multirow{2}{*}{ Критерий } & \multicolumn{2}{|c|}{ 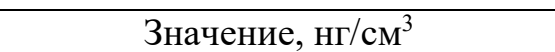 } \\
\hline & Мужчины & Женщины \\
\hline Тестостерон и эпитестостерон & $>200$ & $>50$ \\
\hline Андростерон и этиохоланолон & $>10000$ & $>10000$ \\
\hline $5 \alpha$-андростан- $3 \alpha, 17 \beta$-диол & $>150$ & $>150$ \\
\hline $5 \alpha$-андростан-3 $\alpha, 17 \beta$-диол /эпитестостерон & $>10$ & $>10$ \\
\hline Тестостерон/эпитестостерон & $>4$ & $>4$ \\
\hline Андростерон/тестостерон & $<20$ & $<20$ \\
\hline $\begin{array}{c}5 \alpha \text {-андростан- } 3 \alpha, 17 \beta \text {-диол } / 5 \beta \text {-андростан- } \\
3 \alpha, 17 \beta \text {-диол }\end{array}$ & $>2.4$ & $>2.4$ \\
\hline
\end{tabular}
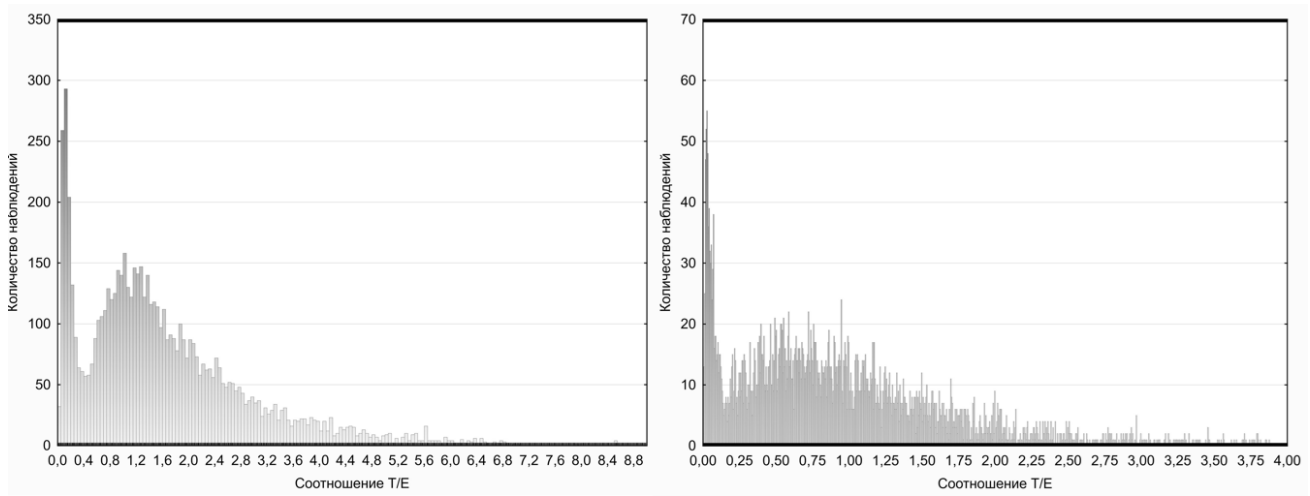

Рис. 1. Наблюдаемые соотношения Т/Е у мужчин (1) и женщин (2).

Fig. 1. Observed ratios for men (1) and women (2).
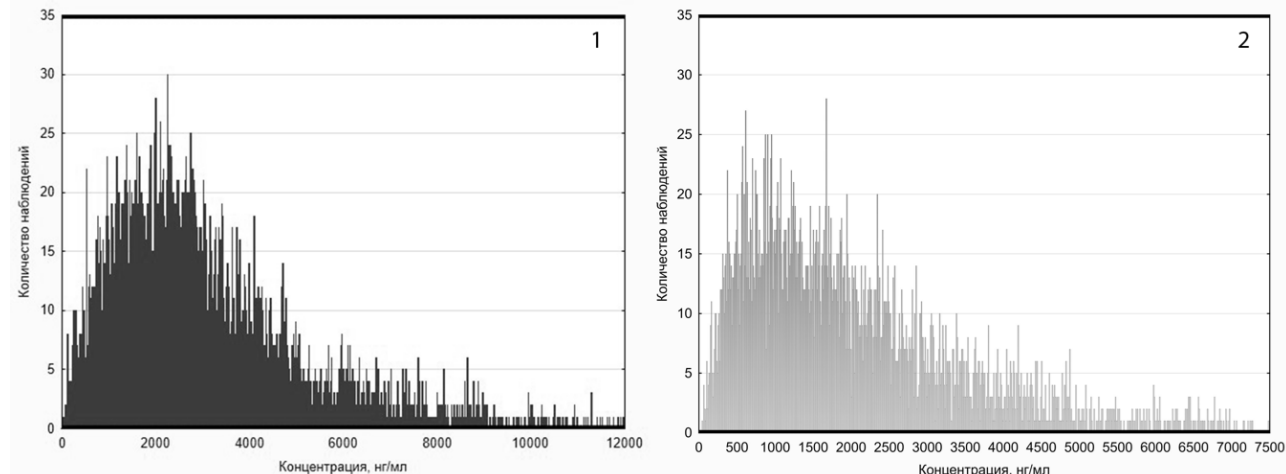

Рис. 2. Наблюдаемые концентрации андростерона у мужчин (1) и женщин (2).

Fig. 2. Observed concentrations of androsterone for men (1) and women (2).

По данным анализируемой выборки, употребление остарина практически не сказывается на стероидном профиле мужчин в отличие от женщин. Основываясь на выборке данных 10 тысяч проб мочи, не содержащих запрещенных ВАДА соединений, предложены более жесткие, по сравнению с ВАДА, критерии (табл. 3), которые являются дополнительным косвенным признаком, свидетельствующем о возможности употребления женщиной препаратов, оказывающих влияние на стероидный профиль, подобно остарину. Выбор критериев проводили из предположения, что 
95\% всех измерений относятся к отрицательным пробам, остальные $5 \%$ считаются подозрительными и нуждаются в дополнительном анализе на наличие соединений, искажающих стероидный профиль.
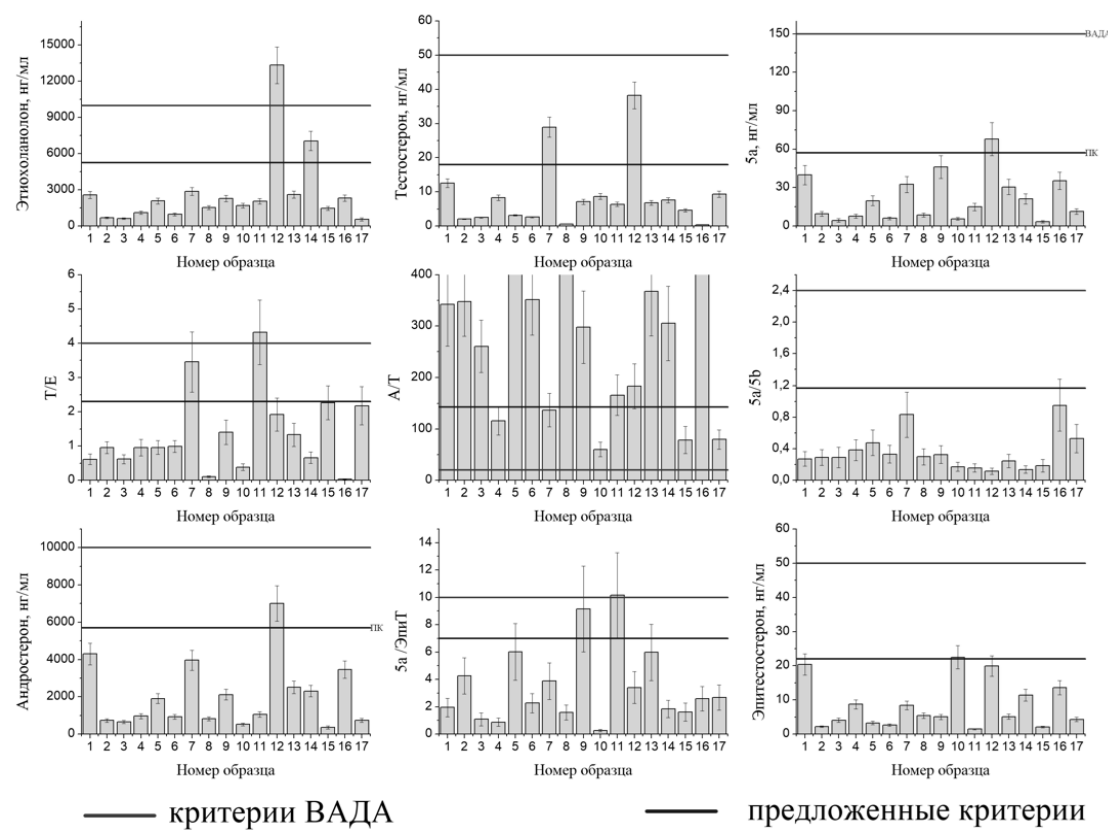

Рис. 3. Наблюдаемые содержания андрогенных стероидов у женщин, в моче которых был обнаружен остарин.

Fig. 3. Observed content of androgenic steroids in women, whose urine contained ostarine.

Таблица 3. Предлагаемые допустимые значения содержания андрогенных стероидов в женской и мужской моче.

Table 3. Suggested permissible content of androgenic steroids in male and female urine.

\begin{tabular}{|c|c|c|}
\hline 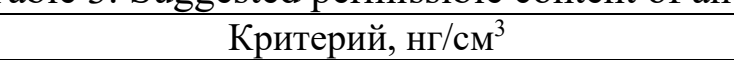 & Женщины & Мужчинь \\
\hline Тестостерон & $>18$ & $>90$ \\
\hline Эпитестостерон & $>22$ & $>70$ \\
\hline Андростерон & $>5700$ & $>7200$ \\
\hline Этиохоланолон & $>5260$ & $>5250$ \\
\hline $5 \alpha$-андростан- $3 \alpha, 17 \beta$-диол & $>57$ & $>159$ \\
\hline 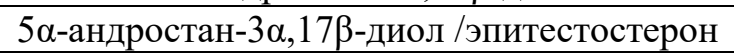 & $>7$ & $>7.8$ \\
\hline Тестостерон/эпитестостерон & $>2.3$ & $>4.2$ \\
\hline Андростерон/тестостерон & $<143$ & $<37$ \\
\hline 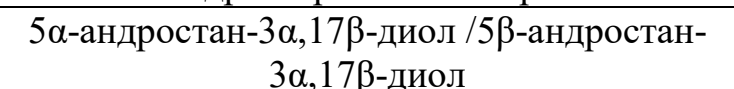 & $>1.17$ & $>1.43$ \\
\hline
\end{tabular}

Использование предложенных критериев позволило корректно выявить 9 из 17 положительных проб на употребление остарина среди женщин на основании косвенных признаков (изменении стероидного профиля). К сожалению, применение аналогичных, более жестких критериев применительно к косвенному обнаружению остарина у мужчин - нецелесообразно, поскольку из 13 положительных проб только 2 незначительно превысили допустимые критерии, при этом одна из проб также превысила менее строгий критерий ВАДА.

Немаловажным фактором является также оценка возникновения ложноположительных результатов после введения более жестких критериев по сравнению с существующими. Отмечено, что ложноположительный результат может возникнуть менее, чем в $1 \%$ рассмотренных случаев, но количество проб с неопределенным ре- 
зультатом (ввиду наличия значимого доверительного интервала у используемой методики) - возрастёт.

Проведя аналогичный анализ положительных проб по экдистерону, было отмечено, что его употребление не приводит к изменению установленных популяционных соотношений, таких, как T/E, или распределение андростерона и не приводит к значительному изменению содержания андрогенных стероидов у мужчин и женщин даже при использовании предложенных, более жестких критериев.

\section{Заключение}

На примере положительных проб с выявленным остарином или его метаболитами показано, что его употребление способно приводить к значимым искажениям стероидного профиля у женщин. Для установления длительности и характера наблюдаемых отклонений требуется курсовое употребление препарата под постоянным контролем медицинского персонала, сопровождающимся регулярным сбором и анализом биологических жидкостей. Отмечено, что употребление экдистерона не приводит к искажению стероидного профиля у мужчин и женщин.

Данная работа выполнена при финансовой поддержке государственного задания Минобрнауки и РФФИ, проект № 19-43-230004 p_a, с использованием научного оборудования Наџиональной антидопинговой лаборатории (институт) Московского государственного университета имени М.В. Ломоносова и ЦКП «Эколого-аналитический цеентр» Кубанского государственного университета.

\section{Список литературы}

1. Davey R.A., Grossmann M. // Clin. Biochem. 2016. Vol. 37. pp. 3-15.

2. McEwan I.J. // Future Med. Chem. 2013. Vol. 5. pp. 1109-1120.

3. Gao W., Dalton J.T. // Drug Discov. Today. 2007. Vol. 12. pp. 241-248.

4. Kim J., Wang R., Veverka K.A., Dalton J.T. // Xenobiotica. 2013. Vol. 43. pp. 9931009.

5. Kintz P., Ameline A., Gheddar L., Raul J.S. // Toxicologie Analytique et Clinique. 2019. Vol. 31. pp. 56-63.

6. de Rijkea E., Essersa M.L., Rijka J.C.W., Thevis M. et al. // Food Addit. Contam. Part A Chem. Anal. Control Expo. Risk Assess. 2013. Vol. 30. pp. 1517-1526.

7. Thevis M., Schänzer W. // Mol. Cell. Endocrinol. 2018. Vol. 464. pp. 34-35.

8. Thevis M., Thomas A., Fusshöller G., Beuck S. et al. // Rapid Commun. Mass Spectrom. 2010. Vol. 24. pp. 2245-2254.

9. Krug O., Thomas A., Beuck S., Schenk I. et al. // Equine. Vet. Sci. 2012. Vol. 32. pp. 562568.

10. WADA 2018 Anti-Doping Testing Figures Report. Режим доступа: https://www.wada- ama.org/sites/default/files/resources/files/2018_ testing_figures_report.pdf (дата обращения 30.05.2020)

11. WADA Athlete Biological Passport Operating Guidelines. Режим доступа: https://www.wada-

ama.org/sites/default/files/resources/files/guidel ines_abp_v71.pdf (дата обращения 30.05.2020)

12. Mohd Rodhia A., Abdul Latif M.N.F., Salima S.A., Abdul Manaf N. et al. // Sci. Sports. 2019. Vol. 34. pp. 52-55.

13.Häkkinen M.R., Heinosalo T., Saarinen N., Linnanen T. et al. // J. Pharm. Biomed. Anal. 2018. Vol. 152. pp. 165-172.

14. Marta Z., Bobaly B., Fekete J., Magda B. et al. // J. Pharm. Biomed. Anal. 2018. Vol. 150. pp. 258-267.

15. De Wilde L., Roels K., Van Renterghem P., Van Eenoo P. et al. // J. Chromatogr. A. 2020 Vol. 1624. pp. 1-10.

16.Iannone M., Palermo A., de la Torre X., Romanelli F. et al. // Steroids. 2019. Vol. 152. 108491. 
17. Saad K., Vonaparti A., Athanasiadou I., Saleh A. et al. // Steroids. 2019. Vol. 152. 108477.

18.Martín-Escudero P., Muñoz-Guerra J.A., García-Tenorio S.V., Garde E.S. et al. // Steroids. 2019. Vol. 141. pp. 104-113.

19. Wang J., Wu M., Liu X., Xu Y. // Steroids. 2011. Vol. 76. pp. 1560-1565.

20.Прасолов И.С. Дисс. канд. хим. наук. М. 2015. $166 \mathrm{c}$.

21.Кочнова Е.А. Дисс. канд. хим. наук. М. 2012. $169 \mathrm{c}$.

22.Tsitsimpikou C., Tsamis G.D., Siskos P.A., Spyridaki M.H. et al. // Rapid Commun. Mass Spectrom. 2001. Vol. 15. pp. 1796-1801.
23.Parr M.K., Ambrosio G., Wuest B., Mazzarino M. et al. // Forensic Toxicol. 2020. Vol. 38. pp. 172-184.

24.Destrez B., Pinel G., Monteau F., Lafont R. et al. // Anal. Chim. Acta. 2009. Vol. 637. pp. 178-184.

25.WADA The 2020 Monitoring Program. Режим доступа: https://www.wadaama.org/sites/default/files/wada_2020_english_ monitoring_program_pdf (дата обращения 30.05.2020)

26.WADA Technical Document TD2015IDCR. Режим доступа: https://www.wadaama.org/sites/default/files/resources/files/td201 5idcr_-_eng.pdf (дата обращения 30.05.2020)

\title{
Chromatographic evaluation of the influence of ostarine and ecdysterone on male and female steroid profiles
}

\author{
(C) 2020 Podolskiy I.I. ${ }^{1}$, Mochalova E.S. ${ }^{2}$, Temerdashev A.Z. ${ }^{3}$ \\ ${ }^{1}$ Bruker Ltd., Moscow, Russian Federation \\ ${ }^{2}$ National Antidoping Laboratory (Institute) of the Lomonosov Moscow State University, \\ Moscow, Russian Federation \\ ${ }^{3}$ Kuban State University, Krasnodar, Russian Federation
}

\begin{abstract}
Selective androgen receptor modulators belong to a new class of doping agents which significantly increase the performance of athletes without the characteristic side effects of anabolic-androgenic steroids. There are now a number of methods used to identify these modulators in human biological fluids. However, there is practically no information about the way they affect the steroid profile. The article describes the influence of ostarine usage on steroid profiles of men and women. The research was based on the qualitative and quantitative analysis of more than 10 thousand urine samples free from WADA prohibited compounds, performed in order to obtain a statistically significant representative sampling, as well as the analysis of 17 female and 13 male ostarine (or its metabolites) positive samples, and 532 male and 245 female ecdysterone positive samples. The quantification of the androgenic steroids was performed by means of gas chromatography (a validated technology) coupled with tandem mass spectrometric detection in the multiple reaction monitoring mode after derivatisation of the samples with the mixture of dithiothreitol, ammonium iodide, and $\mathrm{N}-$ Methyl-N-(trimethylsilyl)trifluoroacetamide. The study demonstrated a significant change in the number of androgenic steroids in female urine after ostarine administration, while in male urine samples this effect was not observed. The use of ecdysterone doesn't significantly affect the content of androgenic steroid hormones in either men or women. Based on the analysed samples that were free from WADA prohibited compounds, we suggest stricter limitations on the content of androgenic steroids for females, which don't lead to a significant increase in the number of false positive results. The approach allows us to obtain additional indirect signs indicating the possibility of an athlete using prohibited substances without resorting to additional procedures and studies. Suspicious or presumably positive samples can be tested in further studies. Such an integrated approach will significantly increase the reliability of studies and enhance the detection of positive samples without increasing the time and cost of the analysis.
\end{abstract}

Keywords: GC-MS/MS, derivatisation, doping, steroids, ostarine, ecdysterone, steroid profile.

\section{References}

1. Davey R.A., Grossmann M., Clin. Biochem., 2016, Vol. 37, pp. 3-15.

2. McEwan I.J., Future Med. Chem., 2013,

3. Gao W., Dalton J.T., Drug Discov. Today, 2007, Vol. 12, pp. 241-248. DOI: 10.1016/j.drudis.2007.01.003 
4. Kim J., Wang R., Veverka K.A., Dalton J.T., Xenobiotica, 2013, Vol. 43, pp. 993-1009. DOI: 10.3109/00498254.2013.788233

5. Kintz P., Ameline A., Gheddar L., Raul J.S., Toxicologie Analytique et Clinique, 2019, Vol. 31, pp. 56-63. DOI: 10.1016/j.toxac.2018.12.001

6. de Rijkea E., Essersa M.L., Rijka J.C.W., Thevis M., Boveea T.F.H. et al., Food Addit. Contam. Part A Chem. Anal. Control Expo. Risk Assess., 2013, Vol. 30, pp. 1517-1526. DOI: 10.1080/19440049.2013.81034

7. Thevis M., Schänzer W., Mol. Cell. Endocrinol., 2018, Vol. 464, pp. 34-35. DOI: 10.1016/j.mce.2017.01.040

8. Thevis M., Thomas A., Fusshöller G., Beuck S. et al., Rapid Commun. Mass Spectrom., 2010, Vol. 24, pp. 2245-2254. DOI: $10.1002 / \mathrm{rcm} .4637$

9. Krug O., Thomas A., Beuck S., Schenk I. et al., Equine. Vet. Sci., 2012, Vol. 32, pp. 562568. DOI: 10.1016/j.jevs.2012.01.005

10. WADA 2018 Anti-Doping Testing Figures Report. Available at: https://www.wadaama.org/sites/default/files/resources/files/2018 testing_figures_report.pdf (accessed 30 May 2020)

11. WADA Athlete Biological Passport Operating Guidelines. Available at: https://www.wada-

ama.org/sites/default/files/resources/files/guidel ines_abp_v71.pdf (accessed 30 May 2020)

12.Mohd Rodhia A., Abdul Latif M.N.F., Salima S.A., Abdul Manaf N. et al., Sci. Sports., 2019, Vol. 34, pp. 52-55. DOI: 10.1016/j.scispo.2018.09.003

13.Häkkinen M.R., Heinosalo T., Saarinen N., Linnanen T. et al., J. Pharm. Biomed. Anal., 2018, Vol. 152, pp. 165-172. DOI: 10.1016/j.jpba.2018.01.034

14.Marta Z., Bobaly B., Fekete J., Magda B., et al., J. Pharm. Biomed. Anal., 2018, Vol. 150, pp. 258-267. DOI: 10.1016/j.jpba.2017.12.014

\footnotetext{
Подольский Илья Игоревич - специалист по применениям хромато-масс-спектрометрического оборудования подразделения Bruker Daltonics, ООО «Брукер», Москва

Мочалова Елена Сергеевна - и.о. директора Национальной антидопинговой лаборатории (институт) Московского государственного университета имени М.В. Ломоносова, Москва

Темердашев Азамат Зауалевич - доцент кафедры аналитической химии, к.х.н., Кубанский государственный университет, Краснодар
}

15. De Wilde L., Roels K., Van Renterghem P., Van Eenoo P. et al., J. Chromatogr. A, 2020, Vol. 1624, pp. 1-10. DOI: 10.1016/j.chroma.2020.461231

16.Iannone M., Palermo A., de la Torre X., Romanelli F. et al., Steroids, 2019, Vol. 152, 108491. DOI: 10.1016/j.steroids.2019.108491

17. Saad K., Vonaparti A., Athanasiadou I., Saleh A., et al., Steroids, 2019, Vol. 152, 108477. DOI: 10.1016/j.steroids.2019.108477.

18.Martín-Escudero P., Muñoz-Guerra J.A., García-Tenorio S.V., Garde E.S. et al., Steroids, 2019, Vol. 141, pp. 104-113. DOI: 10.1016/j.steroids.2018.11.009

19.Wang J., Wu M., Liu X., Xu Y., Steroids, 2011, Vol. 76, pp. 1560-1565. DOI: 10.1016/j.steroids.2011.09.007

20.Prasolov I.S., Diss. cand. him. nauk, Mo., 2015, 166 p.

21.Kochnova E.A. Diss. cand. him. nauk, M., 2012, 169 p.

22.Tsitsimpikou C., Tsamis G.D., Siskos P.A., Spyridaki M.H. et al, Rapid Commun. Mass Spectrom., 2001, Vol. 15, pp. 1796-1801. DOI: $10.1002 / \mathrm{rcm} .433$

23.Parr M.K., Ambrosio G., Wuest B., Mazzarino M. et al., Forensic Toxicol., 2020, Vol. 38, pp. 172-184. DOI: 10.1007/s11419-019-00504$\mathrm{y}$

24.Destrez B., Pinel G., Monteau F., Lafont R. et al., Anal. Chim. Acta, 2009, Vol. 637, pp. 178-184. DOI: $10.1016 /$ j.aca.2008.10.015

25.WADA The 2020 Monitoring Program. Available at: https://www.wadaama.org/sites/default/files/wada_2020_english monitoring_program_pdf (accessed 30 May 2020)

26.WADA Technical Document TD2015IDCR. Available at: https://www.wadaama.org/sites/default/files/resources/files/td201 5idcr_-_eng.pdf (accessed 30 May 2020)

Podolskiy Ilya Igorevich - Application specialist Bruker Daltonics, Moscow, e-mail: podolskiy.i.i@gmail.com

Mochalova Yelena Sergeyevna - Acting director of National Anti-Doping Laboratory (Institute) Lomonosov Moscow State University, Moscow

Temerdashev Azamat Z. - Ph.D. (chemistry), assistant professor, analytical chemistry department, Kuban State University, Krasnodar, e-mail: temerdashevaz@gmail.com 